\title{
NT-proBNP en pacientes con enfermedad pulmonar obstructiva crónica
}

\section{NT-proBNP in chronic obstructive pulmonary disease patients}

\author{
M. Sánchez-Marteles ${ }^{1}$, A. Cecilio-Irazola ${ }^{1}$, D. Vañó-Sanchis ${ }^{2}$, R. Nuviala-Mateo ${ }^{3}$, \\ S. Serrano-Martínez ${ }^{4}$, J.I. Pérez-Calvo ${ }^{1}$
}

\section{RESUMEN}

Introducción. El péptido natriurético cerebral (BNP) es un péptido de producción fundamentalmente ventricular, con múltiples acciones fisiológicas, cuyo principal uso clínico es el diagnóstico y estratificación pronóstica de la insuficiencia cardiaca. El presente trabajo tiene por objeto analizar las concentraciones de NT-proBNP en una población con enfermedad pulmonar obstructiva crónica (EPOC) y su correlación con algunos parámetros que las modifican en condiciones normales.

Material y métodos. Estudio descriptivo, prospectivo, en el que se incluyeron de forma consecutiva pacientes con EPOC ingresados en el servicio de Medicina Interna del H.C.U. "Lozano Blesa" de Zaragoza, del 1 de noviembre de 2004 al 1 de mayo de 2007, por una reagudización de su enfermedad, sin insuficiencia cardiaca y sin criterios de exclusión. Se incluyeron 99 pacientes, a los que se les extrajo sangre en las primeras 72 horas para analizar NT-proBNP.

Resultados. La edad media fue de 74 años, el $79 \%$ fueron hombres. El valor medio del NT-proBNP fue de $1.289 \mathrm{pg} /$ ml. El índice de masa corporal (IMC) medio fue de 27,19. Se encontraron diferencias estadísticamente significativas en las concentraciones de NT-proBNP en relación con la presencia o ausencia de fibrilación auricular y la edad, pero no hubo modificaciones con relación al sexo, IMC o la presencia de insuficiencia renal.

Conclusiones. Los pacientes con EPOC presentan concentraciones elevadas de NT-proBNP durante las reagudizaciones, que se modifican en función de la edad y de la coexistencia o no de fibrilación auricular. Ello podría tener un valor pronóstico, constituyendo una herramienta de selección de los pacientes de riesgo o con peor evolución

Palabras clave. Enfermedad pulmonar obstructiva crónica (EPOC). Insuficiencia cardiaca. BNP.

\begin{abstract}
Background. Brain natriuretic peptide (BNP) is produced and released mainly from ventricles. It has several physiological actions. BNP has been shown to be useful for diagnosis and prognosis in heart failure. The aim of this study is to analyse NT-proBNP levels in chronic obstructive pulmonary disease (COPD) patients and to distinguish factors which could modify these levels.
\end{abstract}

Patients and methods. A descriptive and prospective study was made. COPD patients admitted due to acute exacerbation of this disease at the Hospital Universitario Lozano Blesa (Zaragoza, Spain) were included from November $1^{\text {st }} 2004$ to May $1^{\text {st }} 2007$. We included 99 patients; they had not suffered heart failure and they did not present any exclusion criteria. Blood samples were taken to determine NT-proBNP concentrations.

Results. Mean age was 74 years and $79 \%$ of patients were men. Medium value of NT-proBNP was $1289 \mathrm{pg} /$ $\mathrm{ml}$. Mean body mass index (BMI) was 27.19. There were significant differences between NT-proBNP in patients with or without atrial fibrillation and depending on their age, but there were no differences between men and women nor between patients with or without renal insufficiency.

Conclusion. COPD patients present high serum levels of NT-proBNP during acute exacerbations and these are modified with age and atrial fibrillation. NT-proBNP could be a prognostic factor identifying COPD patients at special risk, or with a worsening clinical evolution.

Key words. Chronic obstructive pulmonary disease (COPD). BNP. Heart failure.
1. Servicio de Medicina Interna. Hospital Clínico Universitario Lozano Blesa. Zaragoza

2. Servicio de Medicina Interna. Hospital General Virgen de la Luz. Cuenca.

3. Servicio de Bioquímica. Hospital Clínico Universitario Lozano Blesa. Zaragoza.

4. Servicio de Bioquímica. Hospital General Virgen de la Luz. Cuenca.

Recepción: 2 de diciembre de 2008

Aceptación provisional: 17 de febrero de 2009

Aceptación definitiva: 8 de abril de 2009

\section{Correspondencia}

Dra. Marta Sánchez-Marteles

Servicio de Medicina Interna

Hospital Clínico Universitario "Lozano Blesa"

Avda. San Juan Bosco, 15

50010 Zaragoza

Tfno: 976765700 (ext 2604)

E-mail: marta.sanchez15@yahoo.es 


\section{INTRODUCCIÓN}

La enfermedad obstructiva crónica (EPOC) es una entidad nosológica frecuente en la práctica clínica diaria, es tratable y tiene una importante repercusión sistémica, así como una no despreciable asociación a otras patologías ${ }^{1,2}$. Su prevalencia en España se cifra en un 9,1\% en edades comprendidas entre los 40 y los 70 años. A nivel mundial es la cuarta causa de mortalidad y su pronóstico está ligado a múltiples factores, entre ellos la comorbilidad asociada ${ }^{1}$.

El péptido natriurético cerebral (BNP) es un péptido de producción fundamentalmente ventricular. Aunque el principal productor es el ventrículo izquierdo, también se ha descrito la síntesis y liberación del péptido a nivel del ventrículo derecho ${ }^{3}$. Su síntesis se ve estimulada ante fenómenos de estrés a dicho nivel, como la sobrecarga o la distensión ${ }^{4}$.

Las acciones fisiológicas son diversas y su principal utilización, hasta la fecha, ha sido el diagnóstico y estratificación pronóstica de la insuficiencia cardiaca. También se ha demostrado su utilidad, especialmente pronóstica, en otras patologías como el tromboembolismo pulmonar (TEP), la hipertensión pulmonar, el cor pulmonale crónico, la cardiopatía isquémica o las valvulopatías ${ }^{4,5,6}$.

La variabilidad del BNP tanto intra como interindividual es importante, habiéndose descrito como factores que modifican sus niveles: la fibrilación auricular, el sexo, la edad, el índice de masa corporal y la insuficiencia renal ${ }^{5}$.

La hipoxemia presente en pacientes EPOC, con frecuencia, implica una vasoconstricción arterial pulmonar que implica, a su vez, un estrés para el ventrículo derecho, con el desarrollo a largo plazo en algunos pacientes de insuficiencia cardiaca derecha ${ }^{7,8}$. Sin embargo, son pocos los estudios que analizan las concentraciones de BNP o su fracción aminoterminal en pacientes con EPOC.

El objetivo de este trabajo es conocer las concentraciones de NTproBNP y la influencia de los factores que habitualmente las modifican, en una población de pacientes con EPOC durante los episodios de descompensación, de cara a conocer futuras utilidades de este biomarcador en este tipo de pacientes.

\section{MATERIAL Y MÉTODOS}

Se trata de un estudio observacional, prospectivo, sin intervención farmacológica, realizado en el servicio de Medicina Interna del Hospital Clínico Universitario "Lozano Blesa" de Zaragoza y el Laboratorio de Análisis Clínicos del Hospital "Virgen de la Luz" de Cuenca.

Se incluyeron de forma consecutiva a todos los pacientes de entre 45 y 85 años, con EPOC y/o bronquitis crónica ingresados en el servicio de Medicina Interna, entre el 1 de noviembre de 2004 y el 1 de mayo de 2007 por una reagudización de su enfermedad respiratoria.

Se consideraron criterios de exclusión la presencia de insuficiencia cardiaca conocida (IC), hipertiroidismo, síndrome de Cushing, tromboembolismo pulmonar (TEP), insuficiencia renal con una creatinina sérica superior a $2 \mathrm{mg} / \mathrm{dl}$, cardiopatía isquémica, valvulopatía mitral y aórtica y neoplasias.

Se consideraron casos prevalentes a todos aquellos pacientes previamente diagnosticados de EPOC, de acuerdo los criterios internacionales establecidos por la Global Initiative for Chronic Obstructive Lung Disease (GOLD) ${ }^{9}$, así como por la Sociedad Española de Neumología y Cirugía Torácica (SEPAR) junto a la asociación Latinoamericana del Tórax (ALAT) ${ }^{1}$, mientras que se consideraron casos incidentes los diagnosticados por primera vez. A efectos del presente estudio se incluyeron los dos tipos de pacientes y consideraron todos en su conjunto.

Todos los casos fueron revisados de manera ciega e independiente por dos médicos internistas expertos para asegurar que cumplían criterios de inclusión y no cumplían criterios clínicos de Framingham ni criterios radiológicos o ecocardiográficos de insuficiencia cardiaca. 
El protocolo seguía las líneas fundamentales de la Declaración Internacional de Helsinki y fue revisado y aprobado por el Comité Ético de la Comunidad Autónoma de Aragón (CEICA), asignándosele el código PI-04/16. Todos los pacientes dieron su consentimiento por escrito previo informe.

A todos los pacientes se les realizó una historia clínica y recogida de datos antropométricos y peristáticos en el momento de su inclusión en el estudio.

La extracción de sangre para la determinación del NTproBNP se realizó en las primeras 72 horas del ingreso, en ayunas y decúbito supino. Las muestras se recogieron en tubos EDTA tripotásico, se centrifugaron, procesaron y almacenaron a $-80{ }^{\circ} \mathrm{C}$ hasta su análisis.

El análisis de todas las muestras se realizó en el Laboratorio de Análisis Clínicos del Hospital "Virgen de la Luz" de Cuenca, utilizando el método Elecsys 2010 (Roche, Inc., Manheim, Germany), con la técnica de inmunoensayo de electroluminiscencia (ECLIA) en analizador Hitachi E170 (Roche).
En el análisis estadístico se realizó un estudio inicialmente descriptivo de las variables. A continuación se estudió su comportamiento mediante el test de Kolmogorov-Smirnov, y de acuerdo a los resultados obtenidos, las comparaciones posteriores se realizaron mediante los test U de MannWhitney y Chi cuadrado. Para ello se utilizó el software SPSS 13.0.

Las variables cualitativas están expresadas en porcentajes (\%), mientras los valores cuantitativos serán expresados mediante la media \pm la desviación típica (DT) o mediana y amplitud intercuartil.

\section{RESULTADOS}

La edad media de los pacientes fue de 74 años (DT: 8 años) y el 79,8\% eran hombres. Más del $75 \%$ de los pacientes tenían una edad por encima de los 65 años.

El 12,1\% tenían niveles séricos de creatinina superiores a $1,2 \mathrm{mg} / \mathrm{dl}$. El IMC medio fue de 27,19 (DT: 4,83), representando los pacientes con sobrepeso u obesidad el $69,7 \%$ de la totalidad de los estudiados. La distribución de la población por edades e IMC se puede apreciar en las figuras 1 y 2 .

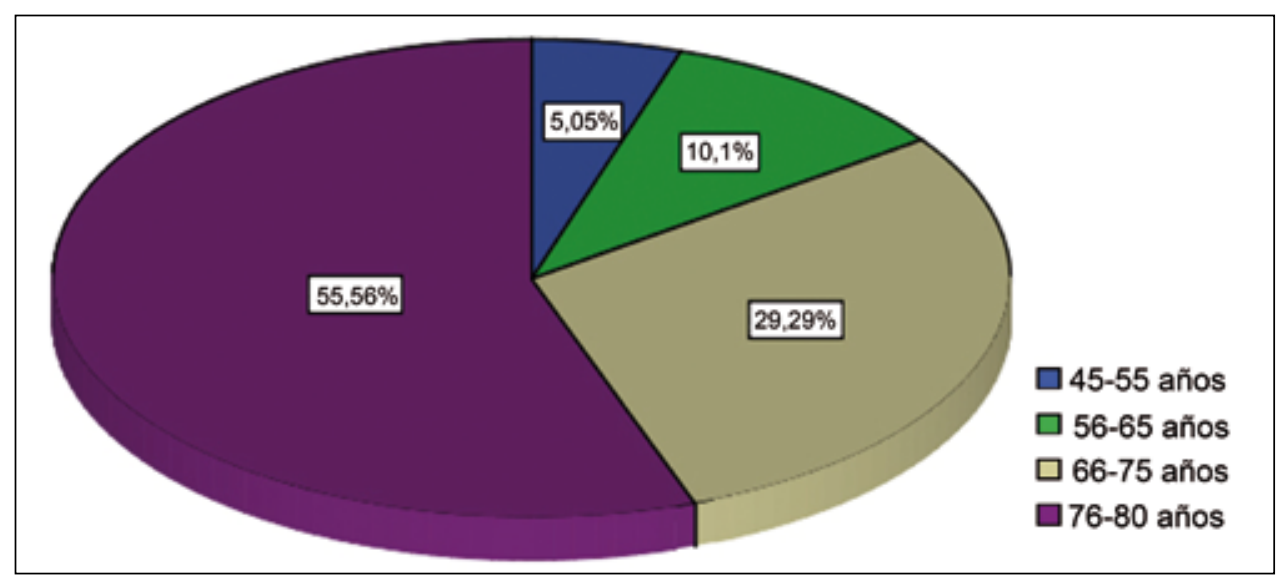

Figura 1. Distribución de los pacientes de la muestra según tramos de edad. 


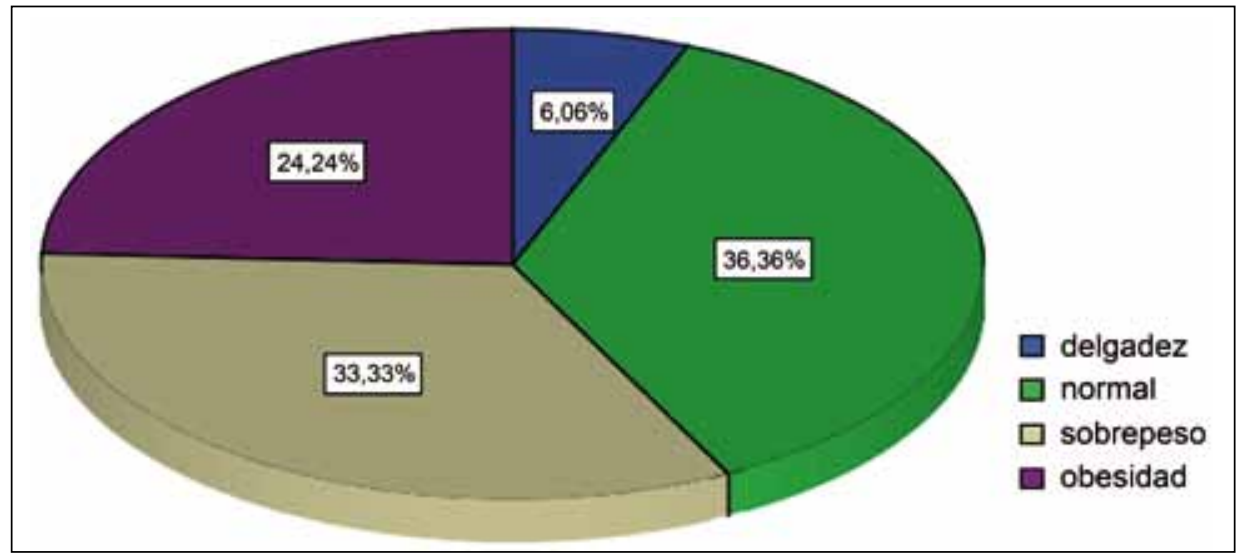

Figura 2. Distribución de los pacientes de la muestra según las categorías de Índice de Masa Corporal (IMC) establecidas por la Organización Mundial de la Salud (OMS).

El valor medio global del NT-proBNP fue de $1.289 \mathrm{pg} / \mathrm{ml}$ (DT: $1.875 \mathrm{pg} / \mathrm{ml}$ ), en los hombres fue de 1.323,95 pg/ml (DT: 1.974 $\mathrm{pg} / \mathrm{ml}$ ) y en mujeres de $1.154 \mathrm{pg} / \mathrm{ml}$ (DT: $1.455 \mathrm{pg} / \mathrm{ml})$.
Los pacientes con fibrilación auricular presentaron concentraciones de NT-proBNP mayores que aquellos otros en ritmo sinusal, siendo las diferencias estadísticamente significativas $(p<0,001$, para $\alpha=0,05$ e intervalo de confianza del 95\%).

Tabla 1. Valores de NT-proBNP en función de los distintos factores analizados.

\begin{tabular}{|c|c|c|c|}
\hline & $\begin{array}{c}\text { Valor medio NT-proBNP (pg/ml) } \\
\text { IC 95\%; DT }\end{array}$ & Mediana (pg/ml) & $\begin{array}{r}\text { Significación } \\
\text { estadística (p) }\end{array}$ \\
\hline $\begin{array}{r}\text { Hombre } \\
\text { Mujer }\end{array}$ & $\begin{array}{l}1.323,95(869,73-1.778,1) ; \text { DT: } 1.974 \\
1.154,7 \text { (453,18-1.856,21); DT: } 1.455,5\end{array}$ & $\begin{array}{l}585,3 \\
574,9\end{array}$ & 0,62 \\
\hline $\begin{array}{l}\text { Edad } \\
\text { (mayor o menor de } 65 \text { años) } \\
\\
>65 \text { años } \\
<65 \text { años }\end{array}$ & $\begin{array}{l}1.470,5(1.018,3-1.922,8) ; \text { DT: } 2.005,9 \\
411,2(158,1-664,4) ; \text { DT: } 418,9\end{array}$ & $\begin{array}{l}260,9 \\
688,8\end{array}$ & 0,002 * \\
\hline $\begin{array}{l}\text { Edad (por décadas) } \\
\qquad \begin{array}{r}45-55 \text { años } \\
56-65 \text { años } \\
66-75 \text { años } \\
76-85 \text { años }\end{array}\end{array}$ & $\begin{array}{l}319,0 \text { (0-729,7); DT: } 258,1 \\
427,7 \text { (98,0-757,3); DT: } 460,8 \\
\text { 1.580,3 (537,0-2.623,7); DT: } 2.637,3 \\
1.377,6 \text { (941,6-1.813,6); DT: } 1.581,8\end{array}$ & $\begin{array}{l}239,2 \\
264,0 \\
621,8 \\
728,6\end{array}$ & $0,02^{*}$ \\
\hline Fibrilación auricular & $\begin{array}{l}1.068,5 \text { (685,2-1.451,8); DT: } 1.688,8 \\
2.698,2(1.300,1-4.096,2) ; \text { DT: } 2.421,3\end{array}$ & $\begin{array}{c}521,6 \\
2.454,0 \\
\end{array}$ & $\mathrm{p}<0,001$ * \\
\hline $\begin{array}{r}\text { Delgadez } \\
\text { Normal } \\
\text { Sobrepeso } \\
\text { Obesidad }\end{array}$ & $\begin{array}{l}1.952,4 \text { (0-5.101,1); DT: } 1.978,7 \\
1.382,98 \text { (-1.196-5.101,1); DT: } 1.978,8 \\
1.476,6(807,3-2145,9) ; \text { DT: } 1.856,4 \\
798,08 \text { (470,8-1125); DT: } 774,9\end{array}$ & $\begin{array}{c}1.544,5 \\
575,4 \\
662,5 \\
563,3\end{array}$ & 0,671 \\
\hline $\begin{array}{l}<1,2 \mathrm{mg} / \mathrm{dl} \\
>1,2 \mathrm{mg} / \mathrm{dl}\end{array}$ & $\begin{array}{l}1.297,6(869,6-1.725,6) ; \text { DT: } 1.947,7 \\
1.235,9(386,9-2.084,8) ; \text { DT: } 1.336,8\end{array}$ & $\begin{array}{l}584,0 \\
684,9\end{array}$ & 0,725 \\
\hline
\end{tabular}

\footnotetext{
* p estadísticamente significativa para $\alpha=5 \% \quad$ Utilizados los Tests U Mann-Whitney y Chi cuadrado

IC: Intervalo de Confianza; DT: Desviación típica
} 
También se observó un aumento progresivo de las concentraciones del péptido en función de la edad. El valor medio de NT-proBNP fue de $319,0 \mathrm{pg} / \mathrm{ml}$ entre los 45 y los 55 años, de $427 \mathrm{pg} / \mathrm{ml}$ entre los $56 \mathrm{y}$ los 65 años, de $1.580 \mathrm{pg} / \mathrm{ml}$ entre los $66 \mathrm{y}$ los 75 años y de $1.377,6 \mathrm{pg} / \mathrm{ml}$ entre los 76 y los 85 años. Las diferencias fueron significativas, tanto categorizando por décadas como agrupando a los pacientes por encima o debajo de los 65 años.

Aunque se observaron concentraciones medias más bajas de NT-proBNP en las mujeres respecto a los hombres, las diferencias en nuestro estudio no fueron significativas $(\mathrm{p}=0,62)$. Tampoco se encontraron diferencias en función del IMC, ni de la función renal hasta un límite de $2 \mathrm{mg} / \mathrm{dl}$ (tomando un punto de corte de creatinina sérica de 1,2 mg/dl (Tabla 1).

\section{DISCUSIÓN}

Como reflejan los informes emitidos por la Sociedad Española de Medicina Interna (SEMI), los pacientes atendidos en los servicios de medicina interna se caracterizan, entre otras cosas por una edad media avanzada e importante pluripatología ${ }^{10}$.

Como se ha descrito en el apartado "Material y Métodos", los criterios de exclusión de nuestro estudio fueron exigentes con el fin de eliminar en la medida de lo posible, sesgos o factores de confusión. Ello implicó, que a pesar de que la EPOC, supone una de las causas más frecuentes de ingreso en estos servicios, un importante porcentaje de pacientes fueron excluidos, lo que limitó el tamaño muestral del estudio, pero aseguró la fiabilidad de sus resultados.

Las concentraciones de péptidos natriuréticos (PN) han demostrado su utilidad clínica en diferentes contextos en los que se produce un estrés mecánico o hemodinámico de la pared del ventrículo izquierdo (VI). Aunque menos conocido, también se sabe que el ventrículo derecho (VD) contribuye a la secreción de $\mathrm{PN}^{3,4}$.

Las reagudizaciones del paciente con EPOC son frecuentes y cursan con hipoxemia, la cual a su vez incrementa las resisten- cias arteriales pulmonares por la vasoconstricción que inducen. Por lo tanto es plausible pensar que en el momento de la reagudización se puede producir un incremento de las concentraciones de PN. Este hecho resulta muy sugestivo a la luz de nuestros resultados, ya que la concentración media de NT-proBNP es de $1.289 \mathrm{pg} / \mathrm{ml}$, superior al punto de corte de exclusión más universalmente admitido en pacientes con IC, que se cifra, de forma general, en $300 \mathrm{pg} / \mathrm{ml}^{11,12}$.

Como ya se ha comentado, el NT-proBNP presenta variaciones en función de distintas características y/o patologías del paciente. Este hecho queda también reflejado en los resultados del presente estudio en el que existe una correlación significativa y positiva entre la edad y la presencia de fibrilación auricular y las concentraciones de NT-proBNP. En cuanto al IMC no existen diferencias significativas entre los distintos grupos, probablemente por el tamaño muestral, si bien existe una gradación apreciable entre los valores extremos (delgadez vs. obesidad).

El valor medio de la concentración de NT-proBNP en nuestro estudio, está por encima de los valores del punto de corte utilizado para el diagnóstico de exclusión de la insuficiencia cardiaca, aun incluyendo una estratificación por edad ${ }^{5,11}$. Son varias las posibles explicaciones que pueden justificar un incremento de la concentración de NT-proBNP en los pacientes EPOC reagudizados. Por un lado, la sobrecarga que genera la hipoxemia sobre el VD, a través de la vasoconstricción pulmonar, puede actuar como un estímulo para la liberación de BNP/NT-proBNP.

Es también posible que las concentraciones elevadas de NT-proBNP de nuestra cohorte de pacientes fueran consecuencia de la existencia de pacientes con disfunción ventricular izquierda subclínica ${ }^{13}$ que hubieran sido mal seleccionados. Creemos que esto puede descartarse razonablemente por cuanto los pacientes incluidos no presentaban signos físicos, ni radiológicos de congestión pulmonar o sistémica y fueron revisados detenidamente por médicos con experiencia y de modo independiente. 
De hecho, se ha demostrado la utilidad de los criterios de Framingham para el diagnóstico de insuficiencia cardiaca, incluso frente al ecocardiograma ${ }^{14}$. Estos criterios tienen una alta sensibilidad en el diagnóstico de insuficiencia cardiaca, con el consiguiente riesgo de diagnóstico de falsos positivos. La consecuencia sobre nuestro estudio, en este caso, es que probablemente se excluyeron pacientes que habrían sido válidos para el mismo.

Por tanto, aunque puede aducirse que algunos pacientes pudieran, en efecto, tener una disfunción VI, esto no afectaría a un grupo suficiente como para condicionar los resultados de la muestra.

Una última explicación es que las concentraciones de NT-proBNP sean debidas a un mayor o menor grado de sobrecarga o disfunción del VD, especialmente, como se ha dicho, en un momento de estrés ventricular secundario a la vasoconstricción hipóxica del lecho pulmonar. La concentración de BNP/NTproBNP ha demostrado su valor pronóstico en la insuficiencia cardiaca. Los descensos significativos de sus concentraciones séricas o plasmáticas en determinaciones seriadas se han asociado a un menor número de reingresos y una menor mortalidad $^{12,15,16}$, lo que permite detectar pacientes que requieren de un seguimiento más estricto o un tratamiento más agresivo. Por otro lado, la hipoxia, el desarrollo de insuficiencia cardiaca y de hipertensión pulmonar son factores pronósticos conocidos en la EPOC ${ }^{1}$. Sea cual sea el mecanismo por el que aumenta la concentración de NT-pro$\mathrm{BNP}$, es plausible que sean representativas de la sobrecarga de presión del VD secundario a la hipoxia y, por lo tanto, que tengan también un valor pronóstico en estos pacientes. De ser esto cierto, dispondríamos de una herramienta más para seleccionar aquellos pacientes con más riesgo de complicaciones o de peor evolución.

Para poder dar respuesta a estas y otras hipótesis relacionadas que se plantean a raíz del presente estudio, son necesarios trabajos prospectivos durante periodos de tiempo más prolongados que están actualmente en marcha.
En conclusión, las concentraciones de NT-proBNP se encuentran elevadas en pacientes con EPOC reagudizada, especialmente en los que tienen FA. Sus valores, por encima del punto de corte de exclusión de insuficiencia cardiaca, sugieren que pueden deberse a grados variables de disfunción VD secundaria a la vasoconstricción hipóxica del lecho pulmonar.

\section{Agradecimientos}

Las determinaciones de NT-proBNP del presente estudio se financiaron mediante una beca de Roche Diagnostics España.

\section{BIBLIOGRAFÍA}

1. Guía de práctica clínica de diagnóstico y tratamiento de la Enfermedad Pulmonar Obstructiva Crónica. SEPAR-ALAT, 2007. Accesible en www.separ.es

2. Wissam MC, Thomashow BM, Minai OA, CRiner GJ, MAKE BJ. Comorbidities in chronic obstructive pulmonary disease. Proc Am Thorac Soc 2008; 5: 549-555.

3. Vogelsang TW, Jensen RJ, Monrad AL, Russ K, Olesen UH, Hesse B, KJAER A. Independent effects of both right and left ventricular function on plasma brain natriuretic peptide. Eur J Heart Fail 2007; 9: 892-896.

4. De Denus S, Pharand C, Williamson DR. Brain natriuretic peptide in the management of heart failure. Chest 2004; 125: 652-668.

5. Almenar-Bonet L, Martínez-Dolz L. Péptidos natriuréticos en insuficiencia cardiaca. Rev Esp Cardiol 2006; 6 Suppl F: 15F-26F.

6. McCullough PA, Omland T, Maisel AS. BType Natriuretic Peptides: A diagnostic breakthrough for clinicians. Rev Cardiovasc Med 2003; Spring 4: 72-80.

7. Stolz D, Breidthardt T, Christ-Crain M, BingisSER R, Miedinger D, LeupPI J et al. Use of B-type natriuretic peptide in the risk stratification of acute exacerbations of COPD. Chest 2008; 133: 1088-1094.

8. BuRKe MA, CotTs WG. Interpretation of B-type natriuretic peptide in cardiac disease and other comorbid conditions. Heart Fail Rev 2007; 12: 23-36.

9. GOLD executive comitee. Global initiative for Chronic Obstructive Pulmonary Disease. Accesible en www.goldcopd.com Visitada en Diciembre de 2008. 
10. Zapatero-Gaviria A, Barba-Martín R. Altas atendidas en Medicina Interna en los hospitales generales del Sistema Nacional de Salud, año 2005. Sociedad Española de Medicina Interna. Grupo de Gestión Clínica de la Sociedad Española de Medicina Interna. Jarpyo ediciones, 2008; 35-39.

11. BAYÉs-Genis A. NT-proBNP circulante, un nuevo biomarcador para el diagnóstico del paciente con disnea aguda. Rev Esp Cardiol 2005; 58: 1142-1144.

12. Januzzi J, Van Kimmenade R, Lainchbury J, Bayes-Genis A, Ordonez-Llanos J, Santalo-Bel M et al. NTproBNP testing for diagnosis and short-term prognosis in acute destabilized heart failure: an international pooled analysis of 1256 patients. The International Collaborative of NTproBNP Study. Eur Heart J 2006; 27: 330-337.

13. Rutten FH, Cramer MJ, Grobbee DE, SAchs AP, KIRKELS JH, LAMMERS JW et al. Unrecognized heart failure in elderly patients with stable chronic obstructive pulmonary disease. Eur Heart J 2005; 26: 1887-1894.

14. Jimeno Sainz A, Gil V, Merino J, García A, Jordán A, Guerrero L. Validez de los criterios de Framingham para el diagnóstico de insuficiencia cardiaca sistólica. Rev Clin Esp 2006; 206 : 495-498.

15. Bettencourt P, Azevedo A, Fonseca L, Araujo JP, Ferreira S, Almeida R et al. Prognosis of decompensated heart failure patients with preserved systolic function is predicted by NTproBNP variations during hospitalization. Int J Cardiol 2007 12; 117: 75-79.

16. Logeart D, Thabut G, Jourdain P, Chavelas C, Beyne B, Beauvais F et al. Predischarge B-type natriuretic peptide assay for identifying patients at high risk of re-admission after decompensated heart failure. J Am Coll Cardiol 2004; 43: 635-641. 
